\title{
Effects of Screen Time and Season on Cardiovascular System Indicators in Primary Schoolchildren
}

\author{
N. B. Pankova ${ }^{a}$ *, I. B. Alchinova ${ }^{a}$, O. I. Kovaleva ${ }^{a}$, M. A. Lebedeva ${ }^{a}$, N. N. Khlebnikova ${ }^{a}$, \\ A. B. Cherepov ${ }^{a}$, L. A. Noskin ${ }^{b}$, and M. Yu. Karganov ${ }^{a}$ \\ ${ }^{a}$ Institute of General Pathology and Pathophysiology, Moscow, Russia \\ ${ }^{b}$ Konstantinov St. Petersburg Institute of Nuclear Physics, St. Petersburg, Russia \\ *e-mail: nbpankova@gmail.com \\ Received February 9, 2021; revised March 17, 2021; accepted March 22, 2021
}

\begin{abstract}
Indicators of the cardiovascular system, including heart rate (HR) and blood pressure (BP) variability parameters, were analyzed in primary school students with different computer screen times. The study included 4084 students of grades 1-4 (age 7-12 years) from 66 Moscow schools. The screen time at school and out of school was assessed by teachers, based on the national Sanitary Rules and Regulations: 0 , no screen time; 1 , screen time matching hygienic standards; 2 , screen time at least twice greater than recommended. Physiological examinations were carried out by spiroarteriocardiorhythmography with a face mask, the conditions corresponding to the functional stress test (mild hypercapnia/hypoxia). Testing took place in spring and autumn (independent samples). Statistical data processing was performed using nonparametric criteria. It was revealed that the introduction of computer technologies in school lessons within the limits of hygienic standards was accompanied by an increase, within the normal range, of systolic BP in girls at the end of grade 2 and 4 and in boys at the beginning and end of grade 4 . Screen time at least twice higher than the hygienic standard did not have an additional effect on BP, but provoked shifts in the function of autonomic regulation. Boys were more sensitive to the influence of this environmental factor. Their pattern of seasonal variability in total power (TP) of the HR variability spectrum was reversed compared to that of children who did not use computers at school; i.e., higher TP values were observed in spring. In grade 4, the process was accompanied by an increase in spontaneous arterial baroreflex sensitivity and a decrease in the relative power of the LF range in the variability spectrum of systolic BP. The changes were assumed to reflect the adaptive response to changes in educational environment.
\end{abstract}

Keywords: blood pressure, heart rate variability, seasonal variability, children, primary school, computer screen time, hygienic standards

DOI: $10.1134 / \mathrm{S} 0362119721060086$

\section{INTRODUCTION}

Assessment of the regulatory systems, including both nervous (autonomic nervous) and humoral (neuroendocrine) systems, is of immense importance for evaluating the functional state of the human body during its growth and development [1]. An informative noninvasive method for the assessment is provided by continuous registration of heart rate (HR) parameters for a few minutes and a subsequent analysis of their variability with algorithms of spectral, geometric, and statistical analyses [2]. HR variability (HRV) is highly efficient in studying the effects of educational environmental factors on the growing body in children. The factors primarily include lack of exercise [3-5] and digital educational technologies, which have come into broadly use in the past years [6, 7].

It is clear that lack of exercise in schoolchildren only increases as computer and information technologies are introduced in the educational environment and reach the current stage of total digital transformation [8]. This was especially clearly seen in the period of online learning due to COVID-19, when the screen time increased almost thrice in schoolchildren $[9,10]$. While a direct negative effect on the cardiovascular system has not been described for using computers and various gadgets [11], a greater portion of sedentary pastimes in a child's behavioral structure increases the risk of cardiometabolic syndrome [12, 13]. In addition, lack of exercise is known to negatively affect the efficiency of the autonomic regulation of the cardiovascular system in children and adolescents [3-5] and to decrease their circadian variability [14].

For many years, we have performed monitoring studies of cardiovascular system parameters in children and adolescents, including analyses of HR and blood pressure (BP) variability indicators by spiroarteriocardiorhythmography (SACR) [15]. Significant changes in parameters were found to occur in Moscow 
first-year primary schoolchildren from 2002-2003 to 2014 [16]: the power of the LF range increased in the HRV spectrum with a respective increase in LF/HF. The sensitivity of the spontaneous arterial baroreflex remained unchanged as established by direct (from measurements in the breathing cycle) and indirect (from the $\alpha$ index) assessments [16]. In addition, seasonal variations were observed for systolic BP (sBP) and the LF/HF ratio in the HRV spectrum [17]. The changes occurring over half a year from spring to autumn and from autumn to spring were studied in primary and secondary school students and adults from 2004 to 2007. We observed that sBP decreased over an academic year and that LF/HF increased. However, opposite processes were detected in primary school students from 2016 to 2019; i.e., sBP increased over an academic year (from autumn to spring) and $\mathrm{LF} / \mathrm{HF}$ decreased (from the second to fifth schooling year) [17].

Thus, substantial changes in cardiovascular system indicators (including their variability parameters, which characterize the state of regulatory systems) were observed in primary school students in the past years. We assumed that the changes are associated with computerization of education. The objective of this work was to check the assumption by analyzing the cardiovascular system indicators (including HRV and BP parameters) in primary school students differing in screen time. The parameters were monitored from 2006 to 2011, when computerization of education just started and new technologies were used in only some schools. Examinations by SACR were carried out in spring and autumn (two independent samples).

\section{METHODS}

We used the data collected at Moscow schools as part of the "Schoolchildren's Health" of the Moscow Health Department (from 2006 to 2011). In total, the data were from 4084 students of grades 1-4 (712 years of age) of 66 various schools; the students lacked verified pathology of the cardiovascular system and had no HR disorders detected in the study.

A SACR hardware and software complex (INTOKS, Russia) simultaneously and continuously records the ECG in standard lead I (with a subsequent HRV analysis), finger blood pressure by photoplethysmography (with a subsequent analysis of sBP and diastolic BP (dBP) variability), and respiratory parameters by using an ultrasonic air flow detector (the subject wears a spirometry mask). Maximal, minimal, and mean values are recorded along with the spectral parameters of HRV and BP (total spectral power (TP) and absolute and relative powers of the standard frequency ranges (HF, LF, and VLF)). It is possible additionally to calculate the indexes based on the spectral variability parameters (LF/HF of the HRV spectrum, centralization index $=(\mathrm{VLF}+\mathrm{LF}) / \mathrm{HF}$ of the HRV spectrum, and $\alpha$ index =
$(\mathrm{LF}(\mathrm{HR}) / \mathrm{LF}(\mathrm{sBP}))^{1 / 2}$ ) and the statistical and geometric variability parameters. Additional options include evaluation of the spontaneous arterial baroreflex sensitivity (using direct measurements in the breathing cycle) and cardiac performance indicators.

All examinations were performed in sitting subjects in the first half of the day. The recording duration was $2 \mathrm{~min}$, thus precluding correct assessment and analysis of the VLF ranges in the HR and BP variability spectra.

The subjects were tested while wearing a spirometry mask and breathing spontaneously. The testing conditions are not indifferent to the subjects [15], and direct measurements of inhaled and exhaled air compositions have confirmed that wearing the mask simulates mild combined hypoxia/hypercapnia [18]. Examination while wearing a spirometry mask has been used as a functional stress test [19].

The screen time at school was estimated by teaches, based on the hygienic norms that were applicable in the study period (Sanitary Rules and Regulations 2.2.2/2.4.1340-03, http://www.consultant.ru/document/cons_doc_LAW_42836/). Screen times were ranked as follows: 0 , no screen time; 1 , screen time matching the sanitary requirements ( $15 \mathrm{~min}$ per day, during a single lesson); and 2, screen time at least twice greater than recommended. Out-of-school screen time was assessed by the teaches by questioning the parents, using the same ranking method.

Examinations were carried out two times per year (in October and from March to April). Examination time points were designated using two digits, of which the first shows the schooling year ( 1 to 4 ) and the second shows the season (1, autumn; 2 , spring). All samples were independent. The sample sizes and general characteristics are summarized in Table 1.

Data distributions were tested for normality by the Shapiro-Wilk test, which allows samples of up to 3000 participants (the Statistica 7.0 package). Based on the testing results, nonparametric tests were used in further statistical analyses. Between-group differences were evaluated by the Kruskal-Wallis $H$-test (multiple comparisons) or Mann-Whitney $U$-test (pairwise comparisons). Associations between indicators were assessed using Spearman's correlation coefficient. Results are presented as the median and interquartile range (Me [Q1; Q3]) in tables and figures.

\section{RESULTS AND DISCUSSION}

The data were generally similar to reference ranges used in medicine (heart rate, BP, and cardiac performance parameters) and reports from other studies (HRV, with due regard to the testing conditions with a spirometry mask on) for gender- and age-matched samples [20]. Significant between-group differences were not observed for respiratory parameters (tidal volume and breathing rate), cardiac performance 
Table 1. Sizes of the children samples examined at different time points

\begin{tabular}{l|r|r|r|r|r|r|r|r}
\hline \multirow{2}{*}{ Testing time point } & \multirow{2}{*}{$n$} & \multicolumn{2}{c|}{ Rank 0 } & \multicolumn{2}{c|}{ Rank 1 } & \multicolumn{3}{c}{ Rank 2 } \\
\cline { 3 - 7 } & & absolute & $\%$ & absolute & $\%$ & absolute & $\%$ \\
\hline $1-1$ & 513 & 94 & 18.3 & 403 & 78.6 & 16 & 3.1 \\
$1-2$ & 310 & 55 & 17.7 & 242 & 78.1 & 13 & 4.2 \\
$2-1$ & 208 & 78 & 37.5 & 122 & 58.7 & 8 & 3.8 \\
$2-2$ & 129 & 68 & 52.7 & 52 & 40.3 & 9 & 7.0 \\
$3-1$ & 332 & 121 & 36.4 & 204 & 61.4 & 7 & 2.1 \\
$3-2$ & 369 & 174 & 47.2 & 180 & 48.8 & 15 & 4.1 \\
$4-1$ & 392 & 126 & 32.1 & 260 & 66.3 & 6 & 1.5 \\
$4-2$ & 350 & 135 & 38.6 & 205 & 58.6 & 10 & 2.9 \\
Total & 2603 & 851 & & 1668 & & 84 & \\
$1-1$ & & & & & & & \\
$1-2$ & 510 & 99 & 19.4 & 395 & 77.5 & 16 & 3.1 \\
$2-1$ & 321 & 69 & 21.5 & 239 & 74.5 & 13 & 4.0 \\
$2-2$ & 96 & 21 & 21.9 & 67 & 69.8 & 8 & 8.3 \\
$3-1$ & 103 & 48 & 46.6 & 49 & 47.6 & 6 & 5.8 \\
$3-2$ & 166 & 15 & 9.0 & 141 & 84.9 & 10 & 6.0 \\
$4-1$ & 129 & 47 & 36.4 & 71 & 55.0 & 11 & 8.5 \\
$4-2$ & 109 & 14 & 12.8 & 90 & 82.6 & 5 & 4.6 \\
Total & 47 & 20 & 42.6 & 22 & 46.8 & 5 & 10.6 \\
\hline
\end{tabular}

Time points are designated with two digits, the first indicating the schooling year ( 1 to 4$)$ and the second, the season (1, autumn; 2, spring).

parameters (stroke volume and cardiac output), spontaneous arterial baroreflex sensitivity (as measured directly in a breathing cycle), and statistical and geometric HRV parameters. As further analyses showed, the most informative parameters in terms of achieving our objective were sBP and the indicators that reflect activity of the autonomic regulation of BP (relative power of the LF range in the sBP spectrum) and HR (TP and the $\alpha$ index, which characterizes the spontaneous arterial baroreflex sensitivity) [21]. Only these indicators showed statistically significant betweengroup differences. Additional attention was paid to LF/HF in the HRV spectrum, because the LF/HF ratio is interpreted as an indicator of autonomic balance $[2,20]$ and shows seasonal variations [17]. Average values of the parameters are summarized in Table 2.

Although independent samples were analyzed in this study, seasonal variation was observed for sBP, which decreased over an academic year from testing time point $1-1$ to point $2-1$ in girls and from point 1-1 to point 4-1 in boys. The changes coincide with the time-related changes that we observed in various age groups from 2004 to 2007. Seasonal variations in other indicators were not confirmed statistically, which is natural because individual variations of the indicators (not only those of the cardiovascular system) are usually rather high and mask the seasonal changes in analyses of average values from independent samples. Repeated measurements in the same sample and evaluation of the differences $(\Delta)$ in the indicators are therefore optimal to use in order to detect seasonal variations $[17,22]$.

Calculation of the coefficients of correlation showed that the indicators chosen for a detailed analysis correlate with the screen time at school, but not with out-of-school screen time (Table 3 ). As is seen from Table 3, a direct correlation between sBP and screen time at school was observed in girls at the ends of the first (point 1-2), second (point 2-2), and fourth (point 4-2) schooling years and in the beginning of the third year (point 3-1) and in boys in the fourth schooling year (points 4-1 and 4-2).

Higher sBP values in schoolchildren who used computers at school in compliance with sanitary regulations as compared with schoolchildren who did not use computers (differences between groups with screen time ranks 0 and 1) were observed in girls at testing time points 2-2 and 4-2 and in boys at time point 4-1 (Fig. 1). In girls, a group with a higher screen 
Table 2. Cardiovascular system indicators (median and interquartile range) in children at different testing time points

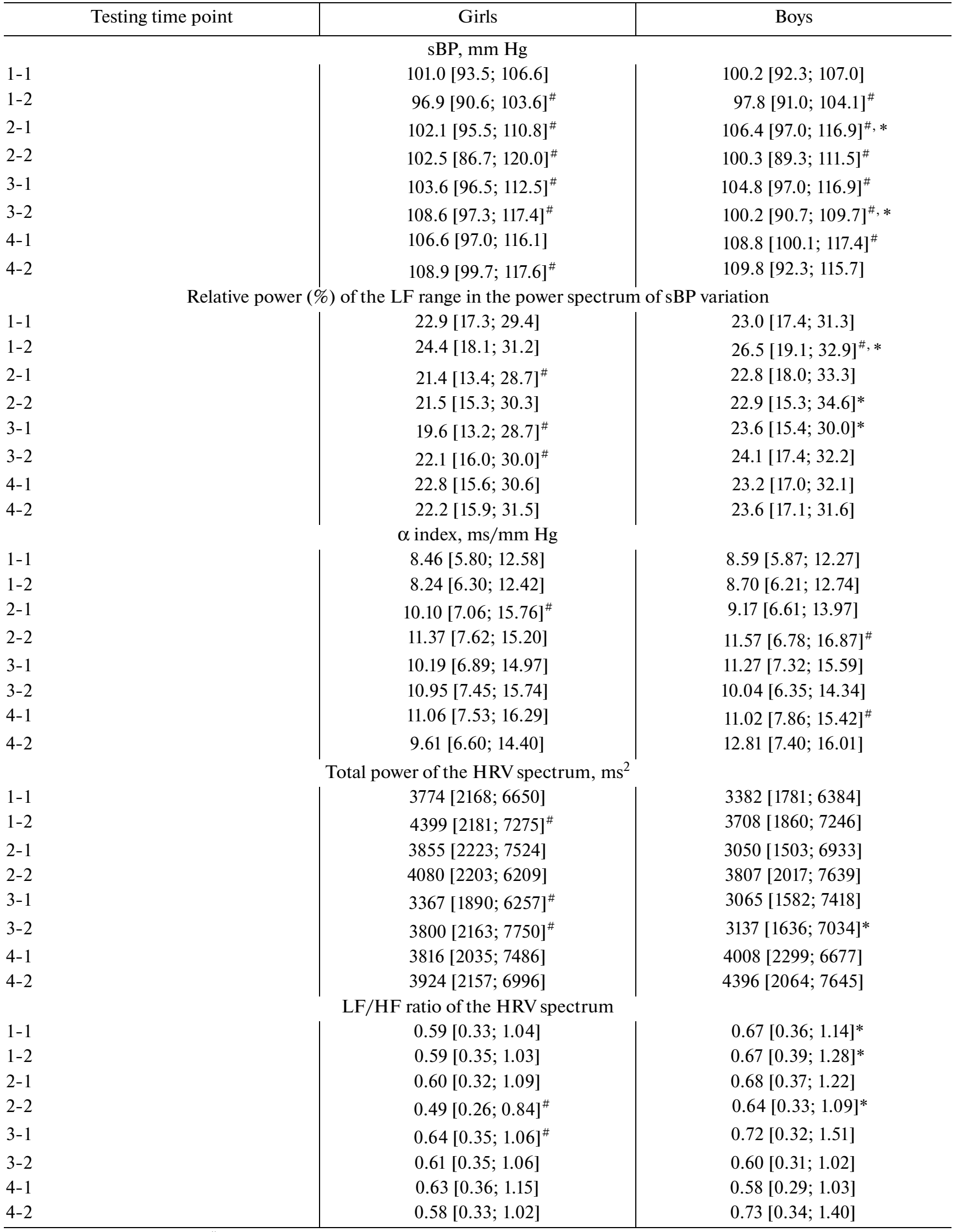

Differences from $(*)$ girls or $\left(^{\#}\right)$ the previous time point were significant at $p<0.05$ by the Mann-Whitney test. 
Table 3. Nonparametric Spearman's coefficients of correlation between the indicators of the cardiovascular system and the screen time at school

\begin{tabular}{|c|c|c|c|c|c|}
\hline Testing time point & sBP & LF\% (sBP) & $\alpha$ index & $\mathrm{TP}$ & $\mathrm{LF} / \mathrm{HF}$ \\
\hline \multicolumn{6}{|c|}{ Girls } \\
\hline $1-1$ & 0.054 & 0.044 & 0.060 & 0.072 & 0.054 \\
\hline $1-2$ & 0.122 & -0.058 & 0.085 & 0.004 & 0.035 \\
\hline $2-1$ & -0.069 & 0.139 & -0.075 & -0.039 & -0.010 \\
\hline $2-2$ & 0.262 & 0.012 & -0.193 & -0.016 & -0.022 \\
\hline $3-1$ & 0.130 & 0.007 & -0.048 & -0.076 & -0.029 \\
\hline $3-2$ & 0.057 & -0.002 & 0.077 & -0.022 & 0.066 \\
\hline $4-1$ & 0.039 & 0.082 & -0.090 & -0.093 & -0.065 \\
\hline $4-2$ & 0.152 & 0.105 & 0.125 & 0.081 & -0.041 \\
\hline \multicolumn{6}{|c|}{ Boys } \\
\hline $1-1$ & -0.121 & -0.032 & 0.023 & 0.056 & 0.003 \\
\hline $1-2$ & 0.004 & 0.059 & 0.032 & -0.054 & 0.085 \\
\hline $2-1$ & 0.019 & -0.097 & -0.126 & -0.063 & 0.056 \\
\hline $2-2$ & 0.085 & 0.178 & -0.038 & 0.044 & -0.035 \\
\hline $3-1$ & 0.035 & 0.101 & -0.093 & -0.144 & 0.079 \\
\hline $3-2$ & 0.089 & 0.117 & -0.050 & -0.061 & 0.157 \\
\hline $4-1$ & 0.238 & 0.055 & -0.254 & -0.222 & 0.057 \\
\hline $4-2$ & 0.331 & -0.469 & 0.419 & 0.319 & -0.451 \\
\hline
\end{tabular}

Statistically significant coefficients $(p<0.05)$ are in bold.

time (rank 2) had lower sBP values as compared with the groups with screen time ranks 0 and 1 in girls at the start of the second schooling year (point 2-1). In contrast, a boy group with rank 2 had higher sBP values at the start of the fourth schooling year (point 4-1).

A normal distribution was observed for a single parameter, sBP, in boys at time points 4-1 and 4-2. The parametric ANOVA algorithm was therefore additionally employed in sBP calculations in the boy groups at the time points. At time point 4-1, $F(2,106)=3.2010, p=0.039$. Differences in mean sBP were evaluated by Tukey's test: $0-1,0.039$ and $0-$ $2,0.235$. The power of the ANOVA test was 1.000 for the three groups with the given mean sBP values and RMSSE calculated to be 14.17 , even when assuming that the three groups are no more than $n=5$ in size. Pairwise comparisons by Student's test were as follows. Comparison 0-1: $\beta=1-0.773=0.227, t=$ $2.466 d f=102, p=0.0153$; comparison $0-2: \beta=1-$ $0.391=0.609, t=1.995 d f=17, p=0.0623$. At time point $4-2, F(2,44)=4.4748, p=0.017$. Differences in mean sBP by Tukey's test: $0-1,0.020 ; 0-2,0.144$. The test power was 1.000 for the three groups with the given mean sBP values and RMSSE calculated to be 10.02 , even when assuming that the three groups are no more than $n=5$ in size. Pairwise comparisons by Student's test were as follows. Comparison $0-1: \beta=1-0.984=$ $0.016, t=2.689 d f=40, p=0.0104$; comparison $0-2$ : $\beta=1-0.819=0.181, t=1.732 d f=23, p=0.0966$.
Thus, in boys examined at testing time points 4-1 and 4-2, group 1 with a screen time meeting the sanitary requirements had a higher mean sBP as compared with group 0 with zero screen time. Group 2 with an elevated screen time tended to differ from group 0 at both time points, but the difference was statistically nonsignificant. However, the high type II error probability makes it possible to assume that the problem is possible to overcome by increasing the size of group 2 .

Russian sanitary requirements are more stringent than the requirements accepted in other countries [23], and the screen times that correspond to rank 2 are comparable with average screen times from similar studies [24]. It is still possible to compare our findings with the results reported by other research teams. There is still no convincing evidence that using computers per se negatively affects the basic parameters of the cardiovascular system [11] and their reactivity in loading tests [7], at least in students. However, additional information loading (associated with using a computer) has been shown to substantially activate the autonomic regulation of the cardiovascular system in 6- to 7-year-old children, shifting the balance towards sympathicotonia [25]. Similar shifts have been described in 9-year-old boys [26] and schoolchildren of the fifth grade [27]. A high screen time (total time at school and at home of more than $2 \mathrm{~h}$ ) increases the risk of hypertension in healthy children and adolescents [28], and the condition is often accompanied by 

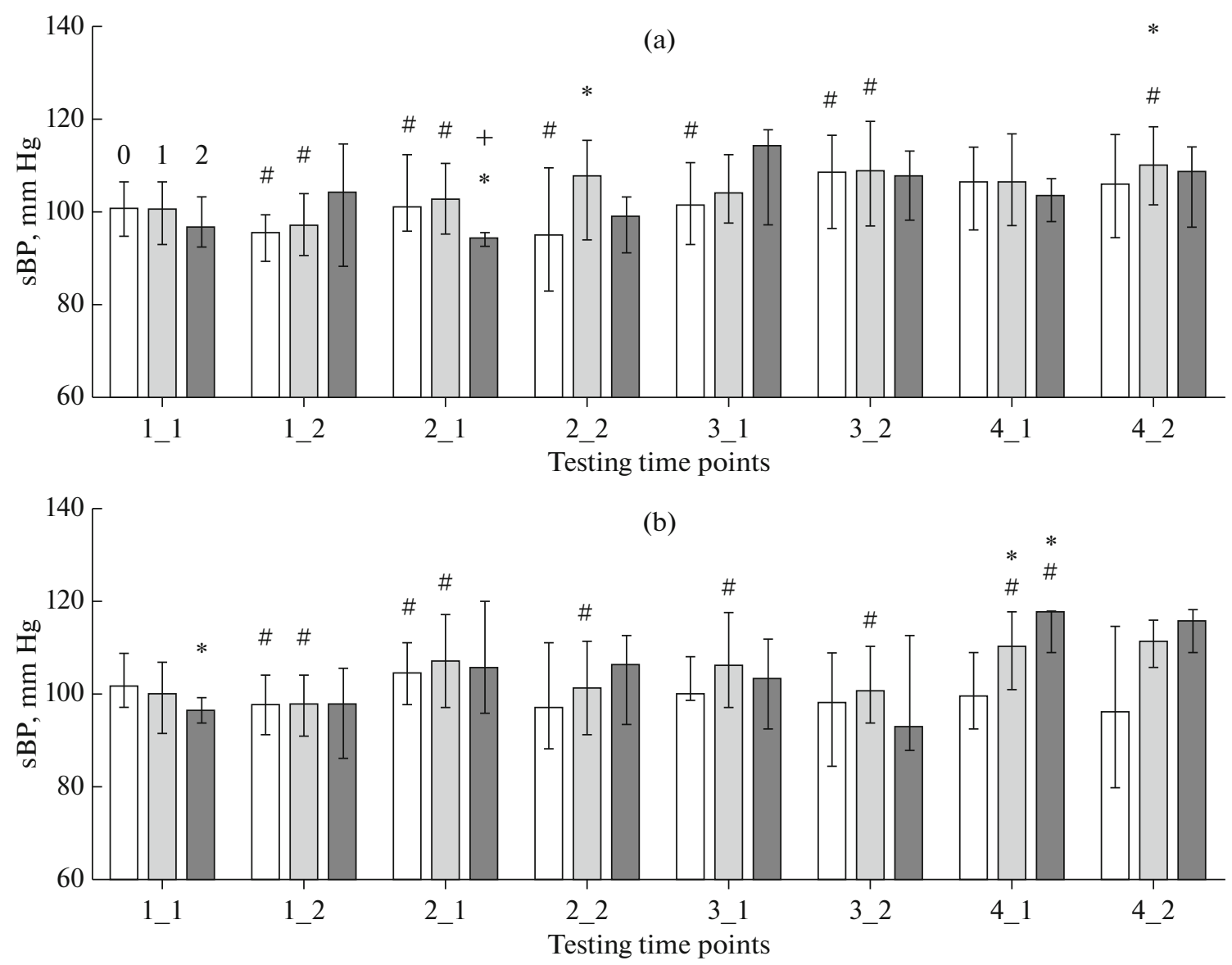

Fig. 1. Systolic BP (sBP, mm Hg; median and interquartile range) in (a) girls and (b) boys. Testing time points are designated with two digits, the first indicating the schooling year and the second, the season (1, autumn; 2, spring). Children groups: 0 , zero screen time; 1 , screen time in accordance with sanitary standards; 2 , screen time at least twice higher than the standard. Statistical significance of between-group differences: $\left({ }^{*}\right)$ difference from group 0 by the Kruskal-Wallis $H$-test $(p<0.05)$, $(+)$ difference from group 1 by the Kruskal-Wallis H-test $(p<0.05)$, and (\#) difference from the previous time point by the MannWhitney $U$-test $(p<0.05)$.

clinical signs of lipid metabolism disorders and a transition to obesity $[29,30]$. An increase in screen time to extreme values in children with internet addiction correlates with autonomic dysfunction (sympathicotonia) of a central origin [31]. Thus, our finding that sBP tends to increase with the introduction of computer technologies into the education environment does not contradict the results reported by other research teams, although the indicator remains within the limits of normal for the gender and age when the sanitary standards are respected.

Computerization of the educational environment is demanding to a child's body [32]. The process acts essentially as a stress factor and induces an adaptive response in children [33, 34]. Traces of the response may change the developmental program of the child's brain and cause morphological alterations in extreme cases [35]. An increase in screen time at an age of 6-7 years decreases the functional potential of the child's body (as revealed using tests for physical development and its autonomic support) [24]. In our study, sBP remained within the limits of normal even in groups with higher screen times (rank 2). We therefore have no reason to think that use of computers in the educational environment is a risk factor to children's health. However, significant shifts in several indicators were observed in the groups with rank 2, reflecting the developing adaptive response. The indicators include not only sBP, but also HR and sBP variability parameters as correlates of the functional state of autonomic regulatory systems. For example, a significant decrease in the relative power of the LF range in the sBP variability spectrum was observed in boys with higher screen times (rank 2) at the end of the fourth schooling year (time point 4-2) in addition to an increase in sBP (Fig. 2). The indicator is usually interpreted as a correlate of the functional activity level of brainstem centers [36] and baroreflex regulation [2, $20]$. We used the $\alpha$ index, which reflects the spontaneous arterial baroreflex sensitivity and is calculated from the spectral parameters of HRV and BP variability [21]. In both boys and girls, higher $\alpha$-index values 


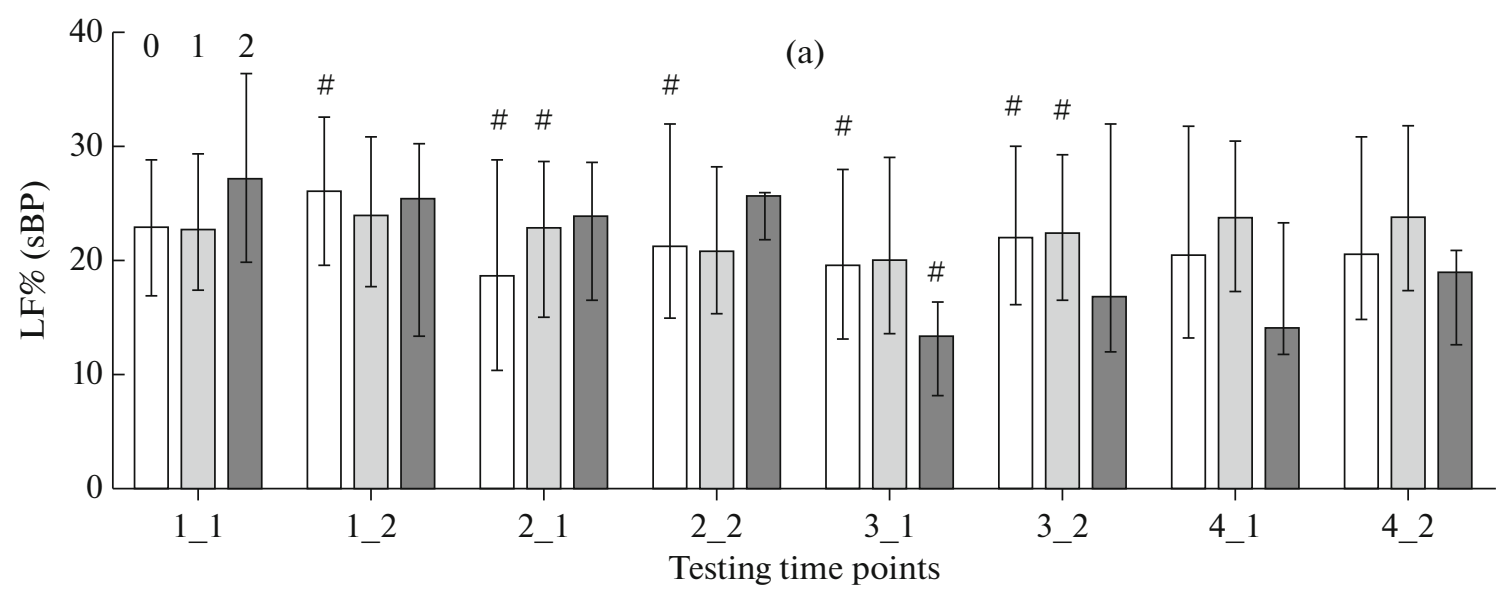

(b)

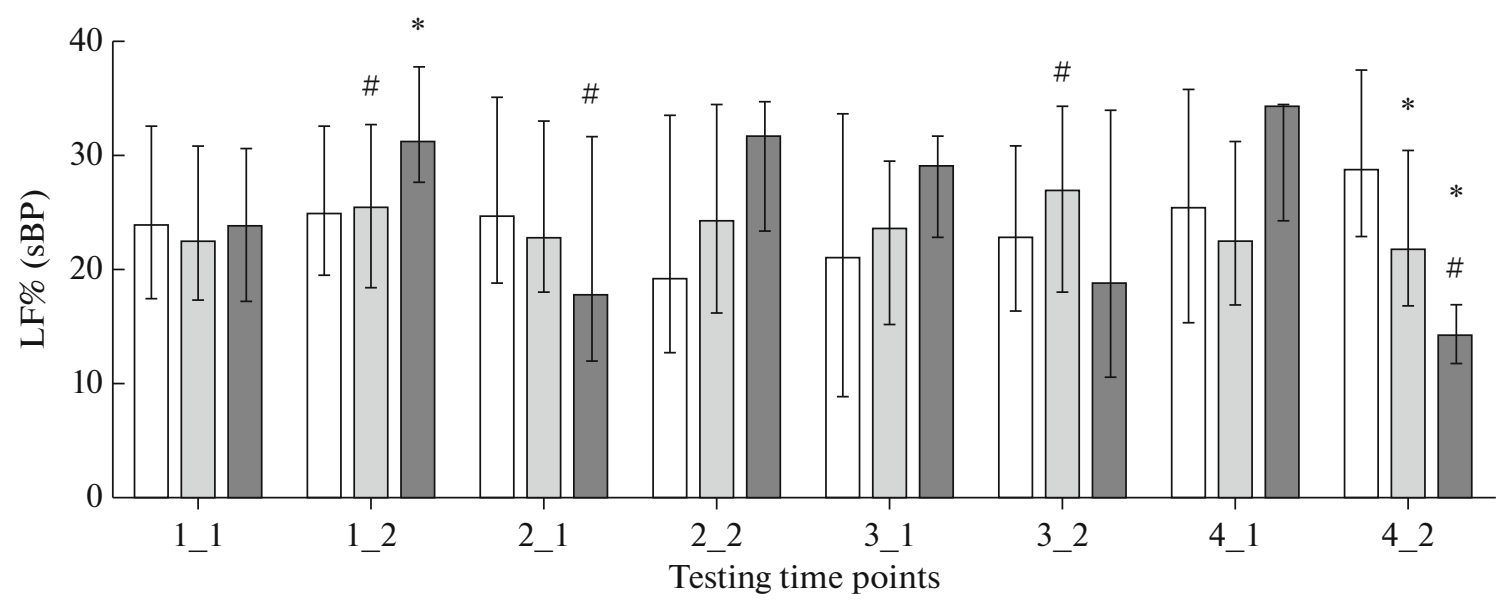

Fig. 2. Relative power (\%, median and interquartile range) of the LF range in the sBP variability spectrum in (a) girls and (b) boys. Designations are as in Fig. 1.

were observed in the groups with higher screen times (rank 2) at time point 4-2 (Fig. 3). There are consequently reasons to assume that higher sBP values may be due to insufficient functional activity of the sympathetic regulation of vascular tone. We did not obtain similar results when analyzing the HRV spectral parameters, but the direction of the above changes coincides with that of changes in LF/HF, which were observed in the monitoring studies from 2016 to 2019 [17].

It should be noted that the $\alpha$ index showed seasonal variations in boys of the third and fourth schooling years and that opposite variations were observed in groups 0 (zero screen time) and 2 (high screen time) (Fig. 3).

Seasonal sBP variation, which manifested itself as a decrease in sBP during an academic year, was characteristic of the pooled sample (Table 2) and reproduced in the groups with rank 0 (zero screen time) within a range from testing time point $1-1$ to point $3-1$ in girls and from point $1-1$ to point $2-1$ in boys. In boys, the same variation pattern was additionally observed in the group with rank 1 (screen time within sanitary standards) in a range from point $1-1$ to point 4-1 (Fig. 1). In girls, an opposite pattern was detected in the group with rank 1; i.e., higher sBP values were observed at the ends of the third and fourth schooling years (points 3-2 and 4-2). The groups with rank 2 (high screen time) did not display seasonal variation of sBP in both girls and boys.

However, a change in the pattern of seasonal variation in autonomic regulation indicators was most clearly seen in the analysis of the TP of the HRV spectrum. The TP is considered to be an integral parameter that reflects activity of the regulatory systems $[2,20]$. In boys, opposite seasonal variations of TP were observed in the groups with ranks 0 (zero screen time) and 2 (high screen time), starting from the second schooling year. TP was higher in autumn in the former and spring in the latter (Fig. 4). In other words, the total level of autonomic activity decreases towards spring in boys learning without using computers, and, oppositely, increases in boys with higher screen times. The process was nonsignificant in girls in our study. 


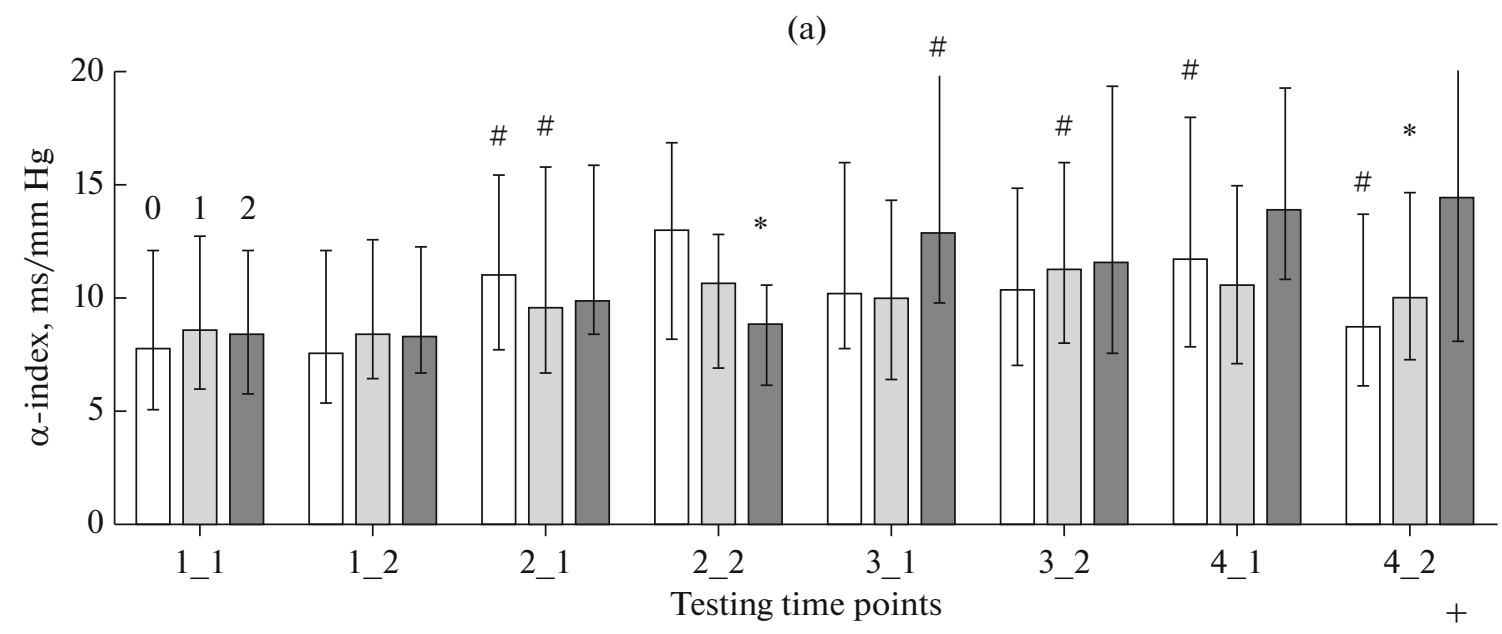

(b)

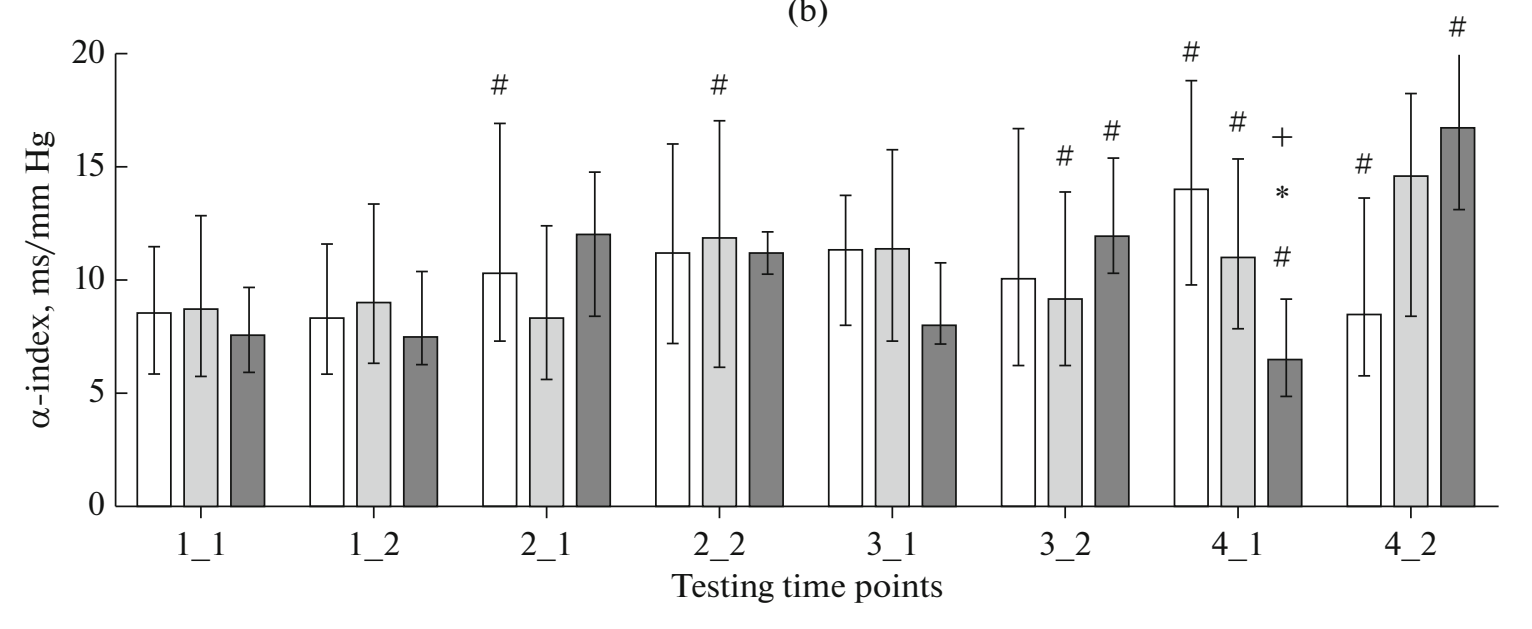

Fig. 3. $\alpha$ index (ms/mm Hg, median and interquartile range) in (a) girls and (b) boys. Designations are as in Fig. 1.

Still opposite seasonal fluctuations of TP in children with different screen times indicate that computerization of the educational environment might be responsible for the change that was observed in the pattern of seasonal variation of cardiovascular indicators when comparing samples tested from 2004 to 2007 with samples tested from 2016 to 2019 [17].

Lack of exercise inevitably accompanies an increase in time that children spend looking at a computer screen or another gadget and certainly acts as an additional factor to induce shifts in the function of autonomic regulatory systems, including metabolic shifts towards anabolism [8-10]. The physical activity level was disregarded in our study. From 2006 to 2011, all Moscow schools followed the same standards with three gym classes per week according to recommendations. Specialized school that focusses on sports training were not included in the study.

It should be emphasized that conditions of a functional stress test [15] were used during testing that revealed the above changes in cardiovascular system indicators in primary school students with different screen times. Such conditions usually provoke a manifestation of latent (preclinical) shifts.

\section{CONCLUSIONS}

Our screening study showed that computerization of education significantly affects the cardiovascular system indicators in primary school students examined in conditions of a functional stress test. In the case where computers were used in the educational environment (in classes at school) within the limits of sanitary standards, sBP increased in girls at the ends of the second and fourth schooling years and boys at the start and end of the fourth schooling year, still remaining within the limits of normal. Screen times at least twice higher than recommended by sanitary standards did not further affect BP, but provoked shifts in the function of autonomic regulatory systems. Boys were more sensitive to this environmental factor. An opposite pattern of seasonal variation in TP of the HRV spectrum was observed in boys with high screen times as compared with boys who did not use a computer at 

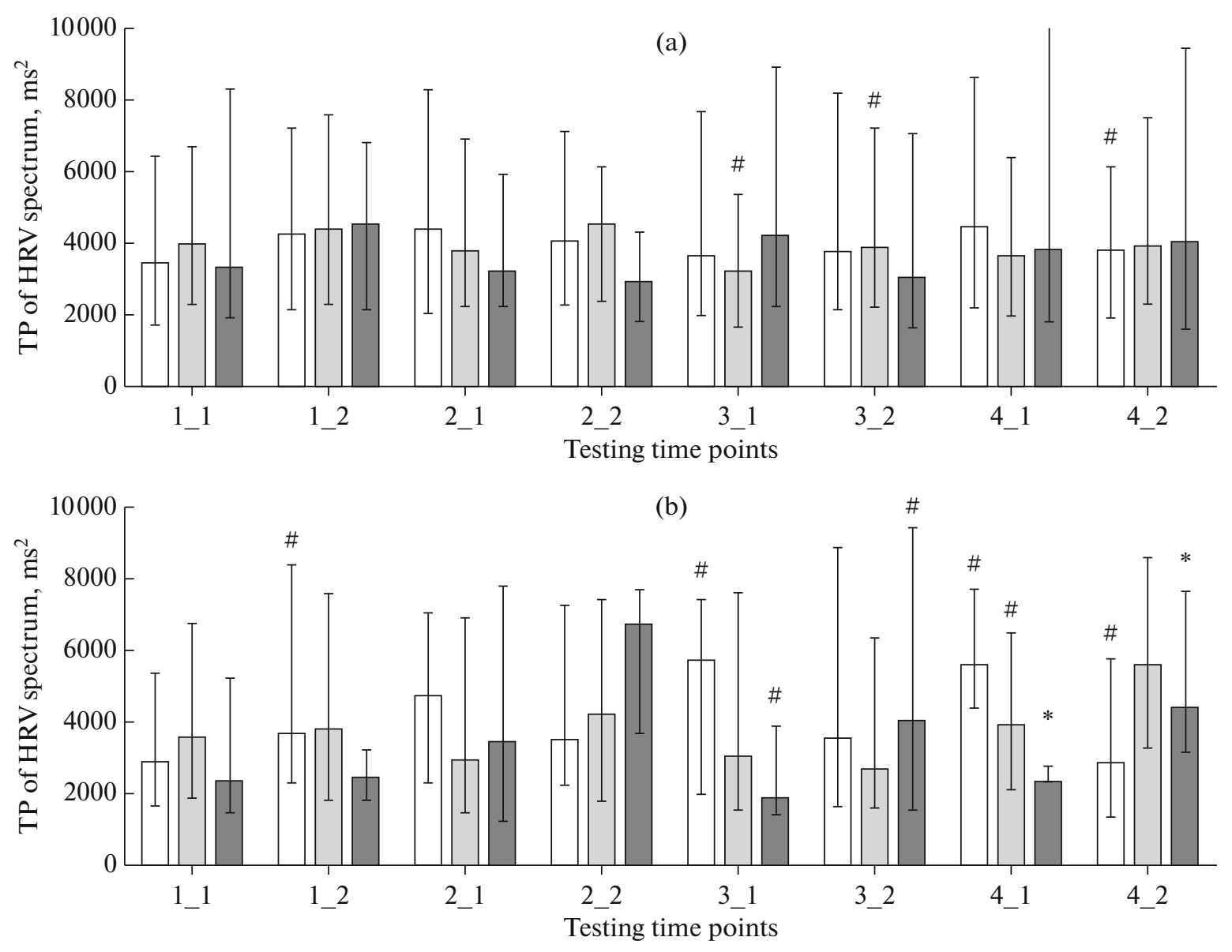

Fig. 4. Total power (TP, $\mathrm{ms}^{2}$; median and interquartile range) of the HRV spectrum in (a) girls and (b) boys. Designations are as in Fig. 1.

school, TP being higher in spring. In the fourth schooling year, the process was accompanied by an increase in spontaneous arterial baroreflex sensitivity and a decrease in the relative power of the LF range in the sBP variability spectrum. The above shifts reflect the adaptive response developing on exposure to changes in educational environment and are certainly beneficial. It is also important to note that the shifts do not go beyond the limits of normal (for the gender and age) even when examination is performed in conditions of a functional stress test.

\section{FUNDING}

This work was supported by the Russian Foundation for Basic Research (project no. 19-29-14104 mk, "Instrumental evaluation of the effect that computerization of education exerts on the physiological balance in the body").

\section{COMPLIANCE WITH ETHICAL STANDARDS}

The study protocol complies with the international (including the Declaration of Helsinki, 2013 revision) and
Russian laws on legal and ethical principls of research involving humans as subjects, as confirmed by the Ethics Committee of the Institute of General Pathology and Pathophysiology, Moscow, Russia (Decision No. 1, 22.01.2019).

\section{INFORMED CONSENT}

In compliance with Articles 5, 6, and 7 of the Universal Declaration of Human Rights, all studies were performed upon informed consent of the students and their parents (or legal representatives).

\section{CONFLICT OF INTEREST}

The authors declare that they have no real or potential conflict of interest.

Statement of compliance with standards of research involving humans as subjects. All procedures performed in studies involving human participants were in accordance with the ethical standards of the 1964 Helsinki Declaration and its later amendments and applicable Russian laws on the legal and ethical principles of research and were approved by the 
Ethics Committee at the Institute of General Pathology and Pathophysiology (Moscow; Minutes no. 1 dated January 22, 2019). In compliance with Articles 5, 6, and 7 of the Universal Declaration on Bioethics and Human Rights, all studies were only performed with consent from students and their parents (or legal representatives).

\section{OPEN ACCESS}

This article is licensed under a Creative Commons Attribution 4.0 International License, which permits use, sharing, adaptation, distribution and reproduction in any medium or format, as long as you give appropriate credit to the original author(s) and the source, provide a link to the Creative Commons license, and indicate if changes were made. The images or other third party material in this article are included in the article's Creative Commons license, unless indicated otherwise in a credit line to the material. If material is not included in the article's Creative Commons license and your intended use is not permitted by statutory regulation or exceeds the permitted use, you will need to obtain permission directly from the copyright holder. To view a copy of this license, visit http://creativecommons.org/licenses/by/4.0/.

\section{REFERENCES}

1. Krivolapchuk, I.A. and Chernova, M.B., Peculiarities of the factorial structure of the functional state in children aged 9-10 years, Hum. Physiol., 2019, vol. 45, no. 1 , p. 30.

2. Baevsky, R.M. and Chernikova, A.G., Heart rate variability analysis: physiological foundations and main methods, Cardiometry, 2017, no. 10, p. 66.

3. Oliveira, R.S., Barker, A.R., Wilkinson, K.M., et al., Is cardiac autonomic function associated with cardiorespiratory fitness and physical activity in children and adolescents? A systematic review of cross-sectional studies, Int. J. Cardiol., 2017, vol. 236, p. 113.

4. Braaksma, P., Stuive, I., Garst, R.M.E., et al., Characteristics of physical activity interventions and effects on cardiorespiratory fitness in children aged 6-12 yearsA systematic review, J. Sci. Med. Sport, 2018, vol. 21, no. 3, p. 296.

5. Veijalainen, A., Haapala, E.A., Väistö, J., et al., Associations of physical activity, sedentary time, and cardiorespiratory fitness with heart rate variability in 6- to 9year-old children: the PANIC study, Eur. J. Appl. Physiol., 2019, vol. 119, nos. 11-12, p. 2487.

6. Makarova, L.V., Lukyanets, G.N., Paranicheva, T.M., and Tyurina, E.V., Effect of computer work on the state of physiological functions in children aged 7 to 10 years, Hum. Physiol., 2017, vol. 43, no. 2, p. 177.

7. Kourova, O.G., Popova, T.V., Kokoreva, E.G., et al., Ecological and physiological aspects of use of computer technologies in educational process, Ekol. Chel., 2019, no. 7, p. 59.

8. Carson, V., Hunter, S., Kuzik, N., et al., Systematic review of sedentary behavior and health indicators in school-aged children and youth: an update, Appl. Physiol. Nutr. Metab., 2016, vol. 41, no. 6-3, p. S240.
9. Kuchma, V.R., Sedova, A.S., Stepanova, M.I., et al., Features of the life and well-being of children and adolescents during remote studing due to the epidemic of a new coronavirus infection (COVID-19), Vopr. Shk. Univ. Med. Zdorov'ya, 2020, no. 2, p. 4.

10. Xiang, M., Zhang, Z., and Kuwahara, K., Impact of COVID-19 pandemic on children and adolescents' lifestyle behavior larger than expected, Prog. Cardiovasc. Dis., 2020, vol. 63, no. 4, p. 531.

11. Stiglic, N. and Viner, R.M., Effects of screen time on the health and well-being of children and adolescents: a systematic review of reviews, BMJ Open., 2019, vol. 3, no. 9, p. e023191.

12. Vanderloo, L.M., Keown-Stoneman, C.D.G., Sivanesan, H., et al., Association of screen time and cardiometabolic risk in school-aged children, Prev. Med. Rep., 2020, vol. 20, p. 101183.

13. Sivanesan, H., Vanderloo, L.M., KeownStoneman, C.D.G., et al., The association between screen time and cardiometabolic risk in young children, Int. J. Behav. Nutr. Phys. Act., 2020, vol. 17, no. 1, p. 41.

14. Miyagi, R., Sasawaki, Y., and Shiotani, H., The influence of short-term sedentary behavior on circadian rhythm of heart rate and heart rate variability, Chronobiol. Int., 2019, vol. 36, no. 3, p. 374.

15. Trukhanov, A.I., Pankova, N.B., Khlebnikova, N.N., and Karganov, M.Yu., The use of spiroarteriocardiorhythmography as a functional test for estimating the state of the cardiorespiratory system in adults and children, Hum. Physiol., 2007, vol. 33, no. 5, p. 585.

16. Pankova, N.B. and Karganov, M.Yu., Comparative analysis of indicators of the functional state in contemporary Moscow first-graders and their peers in 20022003, Vestn. Novosib. Gos. Pedagog. Univ., 2017, vol. 7 , no. 1, p. 173.

17. Pankova, N.B. and Karganov, M.Yu., Seasonal and secular variations in selected indicators of the cardiovascular system among 7-11 years old children, Ekol. Chel., 2020, no. 12, p. 3.

18. Pankova, N.B., Arkhipova, E.N., Alchinova, I.B., et al., Comparative analysis of rapid assessment methods of the functional state of the cardiovascular system, Vestn. Vosstanovitel'noi Med., 2011, no. 6 (46), p. 60.

19. Pankova, N.B., Mavrenkova, P.V., Lebedeva, M.A., and Karganov, M.Yu., Indicators of heart rate variability and blood pressure in adolescents with neuropsychiatric disorders during exercise tests, Patogenez, 2020, vol. 18 , no. 4, p. 64.

20. Shaffer, F. and Ginsberg, J.P., An overview of heart rate variability metrics and norms, Front. Publ. Health, 2017, vol. 5, p. 258.

21. Rydlewska, A., Ponikowska, B., Borodulin-Nadzieja, L., et al., Assessment of the functioning of autonomic nervous system in the context of cardiorespiratory reflex control, Kardiol. Pol., 2010, vol. 68, no. 8, p. 951.

22. Bhutani, S., Hanrahan, L.P., Vanwormer, J., and Schoeller, D.A., Circannual variation in relative weight of children 5 to 16 years of age, Pediatr. Obes., 2018, vol. 13 , no. 7 , p. 399.

23. Gottschalk, F., Impacts of Technology Use on Children: Exploring Literature on the Brain, Cognition and Well- 
Being: OECD Education Working Papers No. 195, Paris: Org. Econ. Coop. Dev., 2019.

24. Krivolapchuk, I.A., Gerasimova, F.F., and Chernova, M.B., Functional development of 6-7years old children with different levels of informatization of living conditions, Sib. Pedagog. Zh., 2020, no. 5, p. 121.

25. Krivolapchuk, I.A. and Chernova, M.B., Schoolchildren's functional state under intensive information load at the initial adaptation period to educational environment, Ekol. Chel., 2018, no. 9, p. 18.

26. Litvin, F.B., Bruk, T.M., Terekhov, P.A., and Bykova, I.V., Dynamics of heart rate variability during the school year in 8-9-year-old boys from different radioecological places of residence, Kul't. Fiz. Zdorov'e, 2019, vol. 72, no. 4, p. 152.

27. Chetverik, O.N., Tarasova, O.L., and Kazin, E.M., Psychophysiological adaptation of fifth-year school graduates to different modes of the educational process, Psikhol., Psikhofiziol., 2019, vol. 12, no. 2, p. 89.

28. Wyszyńska, J., Podgórska-Bednarz, J., Dereń, K., and Mazur, A., The relationship between physical activity and screen time with the risk of hypertension in children and adolescents with intellectual disability, Biomed. Res. Int., 2017, vol. 2017, p. 1940602.

29. Heshmat, R., Qorbani, M., Babaki, A.E.S., et al., Joint association of screen time and physical activity with cardiometabolic risk factors in a national sample of Iranian adolescents: the CASPIAN III study, PLoS One, 2016, vol. 11, no. 5, p. e0154502.
30. Norman, G.J., Carlson, J.A., Patrick, K., et al., Sedentary behavior and cardiometabolic health associations in obese 11-13-year olds, Child Obes., 2017, vol. 13, no. 5 , p. 425 .

31. Lin, P.-C., Kuo, S.-Y., Lee, P.-H., et al., Effects of internet addiction on heart rate variability in school-aged children, J. Cardiovasc. Nurs., 2014, vol. 29, no. 6, p. 493.

32. Baiguzhin, P.A., Shibkova, D.Z., and Aizman, R.I., Factors affecting the psychophysiological processes of perception of information in the conditions of informatization of the educational environment, Sci. Educ. Today, 2019, vol. 9, no. 5, p. 48.

33. Gunnar, M. and Quevedo, K., The neurobiology of stress and development, Annu. Rev. Psychol., 2007, vol. 58 , no. 1 , p. 145.

34. O'Connor, D.B., Thayer, J.F., and Vedhara, K., Stress and health: a review of psychobiological processes, $A n-$ nu. Rev. Psychol., 2021, vol. 4, no. 72, p. 663.

35. Hoffman, E.A., Clark, D.B., Orendain, N., et al., Stress exposures, neurodevelopment and health measures in the ABCD study, Neurobiol. Stress, 2019, vol. 10, p. 100157.

36. Pfurtscheller, G., Schwerdtfeger, A.R., Rassler, B., et al., Verification of a central pacemaker in brain stem by phase-coupling analysis between HR interval- and BOLD-oscillations in the $0.10-0.15 \mathrm{~Hz}$ frequency band, Front. Neurosci., 2020, vol. 14, p. 922.

Translated by T. Tkacheva 